\title{
The Decision Support System of Urban Distribution Network Planning Based on Electric Power Demand Forecasting Suling Chen
}

\author{
Henan Electric Power Survey \& Design Institute, Zhengzhou, 450000, China \\ email: cslhnu@163.com \\ hunter2011@foxmail.com
}

Keywords: Distribution Network Planning; Power Demand Forecasting; Decision support system

\begin{abstract}
The functional architecture and overall design of the decision support system of urban distribution network planning based on electric power demand forecasting are introduced, with the focus on the function implementation and key planning results of the electric power demand forecasting module for the system. Based on the actual planning operation of $\mathrm{H} \mathrm{City,} \mathrm{this} \mathrm{system}$ employs the latest computer technology and technical expertise for optimization and management of distribution network planning in H City. Compared with the traditional planning software, this system enjoys higher technology practical levels, and thus provides a basis for the informatization and refined management of distribution network planning.
\end{abstract}

\section{Introduction}

Power grid construction is an important part of power system construction, its task is to build scientific and rational power grid structure according to the load growth, and to make the grid construction and operating costs minimized under the premise of transferring power to the load center economically, reliably and safely. Network planning is not only an important part of the national economy and social development in the supply region, but also an important basis for the long-term development plan of the power grid corporation. The target of network planning is to make the grid development able to meet the requirements of the economy moderately ahead in the supply region, and play a leading and decisive part in grid construction, operation and supply security[1-2].

The decision support system of urban distribution network planning based on electric power demand forecasting (hereinafter referred to as the "system"), which mainly makes use of the existing data collected from SCADA, EMS and other systems, employs computer technology and artificial intelligence technology to complete the analysis of the situation, electric power demand forecasting, power source structure optimization, power supply planning of important user, substation location planning, project investment estimation and investment benefit evaluation. System employs a visual interface for intelligent operations management, in order to improve the level of intelligence, automation and visualization of the network planning.

\section{System Design}

Urban distribution network planning is a complicated engineering system, also a day-to-day work; process is shown in figure1. System is designed according to the miscellaneous function of the various distribution networks planning process.

Based on computer technology, network communication, information processing and safety management mode, system takes power equipment management as foundation, visualization of function module as interface, power demand forecasting as core. Through the management of regional power grid and its power equipment, the system makes auxiliary analyses of medium and low voltage power distribution network planning, investment estimation and benefit assessment of power distribution project, in order to standardize the management of the distribution network planning, rationalize the arrangement of power grid operation, optimize the planning. In addition, 
system is able to improve the efficiency of urban distribution network planning management work greatly by the employment of visual interface.

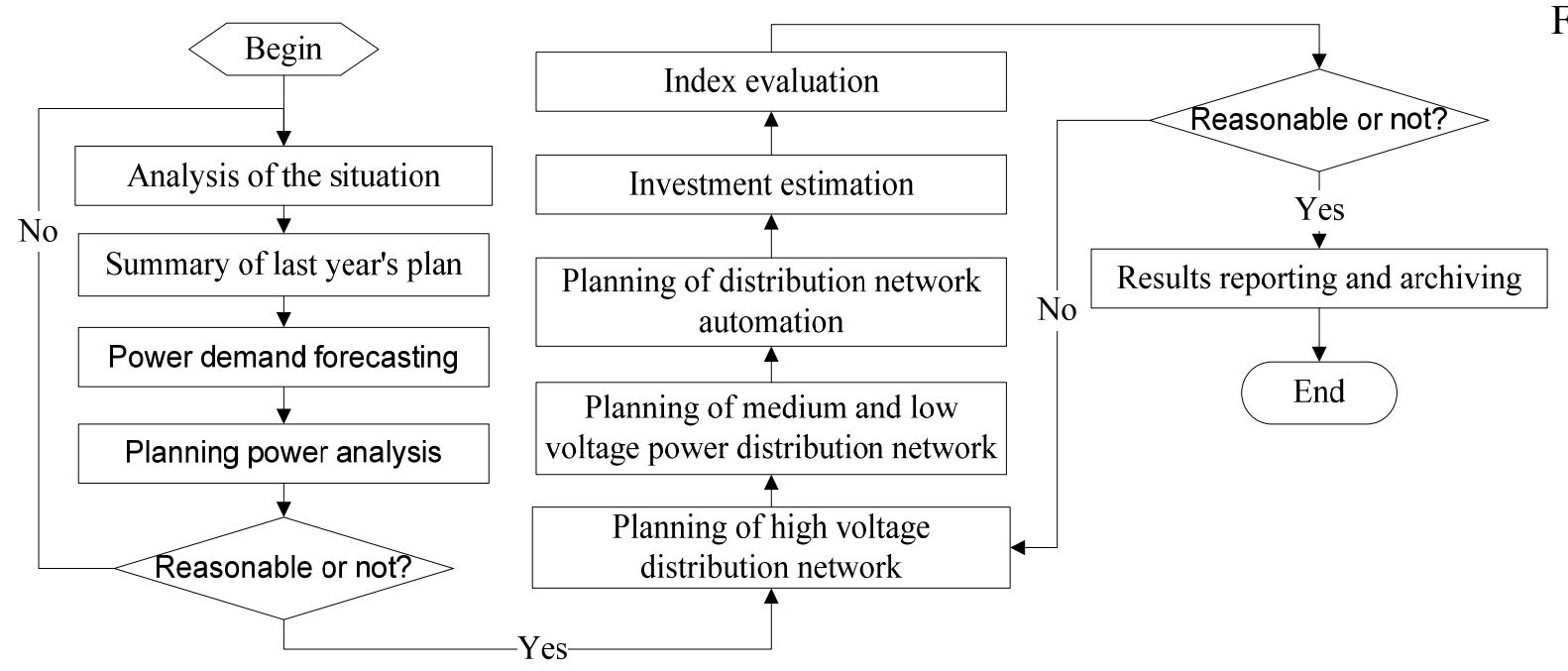

Fig

Fig. 1. Process of Distribution Network Planning

Design platform: the system is mainly based on J2EE architecture of My Eclipse platform, employs Java language and XML programming. The Java language is well adaptable; it is able to run independently in various servers. Moreover, Good class encapsulation, convenient code maintenance of the Java language makes the system transplanted and maintained well.

Database: oracle10g is one of the most popular databases currently. It is able to handle huge amounts of data faster than other databases with less resources occupation, and it is also great improved in management and security. Oracle $10 \mathrm{~g}$ provides a new distributed database capacity, which makes reading and writing more easily in the remote database through the network.

$\mathrm{B} / \mathrm{S}$ (Browser/Server) structure: compared with $\mathrm{C} / \mathrm{S}$ (client/server) mode, the $\mathrm{B} / \mathrm{S}$ structure holds the following advantages: firstly, system level is distinct with the $\mathrm{B} / \mathrm{S}$ mode separating logic, data and client layer; secondly, the B/S mode only needs to install the developed system on the server side, so system can be accessed directly in the form of web pages.

\section{System Module}

The system is divided into the following eight modules.

(1) My home page. Different users in this module can receive and release, upload results, complete the internal communication, which is convenient for distribution network planning.

(2) Analysis of the situation. This module employs reports and charts tools to establish the analysis system of urban distribution network situation, thus to provide better technical support in improving the level of planning project decisions and the usage efficiency of funds.

(3) Power demand forecasting. This module is a core part of the system; it mainly implements integrated management of various forecasting methods. It is able to choose optimal forecast model according to the forecasting environment characteristics and data characteristics, to complete layered, partitioned and different dimensional load forecasting, and finally implement mutual check and linkage adjustment of forecasting results obtained by different ways.

(4) High voltage distribution network planning. On the basis of the power demand forecasting, this module mainly employs computer technology to determine the location and capacity of substations to build in the target and the middle years automatically, in order to meet the needs of the future load development. Under the condition of minimizing investment and operating cost, this module is able to calculate power supply scope of substations and load rate of main transformer automatically, and display substations location as well as the outcome of the grid network planning visually.

(5) Middle and low voltage distribution network planning. According to the results of power 
demand forecasting, the location of the substation, as well as the road network of municipal planning, this module mainly determines the trend of $10 \mathrm{kV}$ trunk lines, the location and number of distribution equipment such as switching station, ring main unit, etc. By combining reconstruction with new construction and putting the most urgent at the top of a list of matters to deal with, it solves the medium voltage power grid status problems standardly, such as heavy load and overload medium voltage circuit, the unqualified terminal voltage, safety hidden danger, medium voltage network frame, etc. And it solves the low voltage power grid status problems such as heavy load and overload distribution transformers, lower area voltage, prolonged power supply radius, new load, unsafe lines and equipment, travel-weary equipment, etc.

(6) Distribution network automation planning. This module mainly implements statistics and summary of urban distribution network automation projects and special projects.

(7) Investment estimation and economic evaluation. This module employs reports and charts tools to count investment year by year and classified of investment, in order to analyze the rationality of planning investment.

(8) Planning evaluation [3]. The module mainly employs reports and charts to analyze the solution rate of distribution network problems, and the promotion effect of power supply reliability, as well as the reduction of line loss rate which distribution network planning schemes contribute to. It is able to judge the rationality of the planning scheme through the comparison of status indicators with indicators after planning scheme.

\section{Design of Power Demand Forecasting Module}

Power demand forecasting including electric quantity forecasting, peak load forecasting and spatial load forecasting, is the premise and basis of power distribution network planning. The accuracy of the forecasting is directly related to the quality of the planning schemes, the safe and economic operation of distribution network. Compared with traditional forecasting software which pays more attention to the algorithm model, the system enjoys higher technology practical levels.

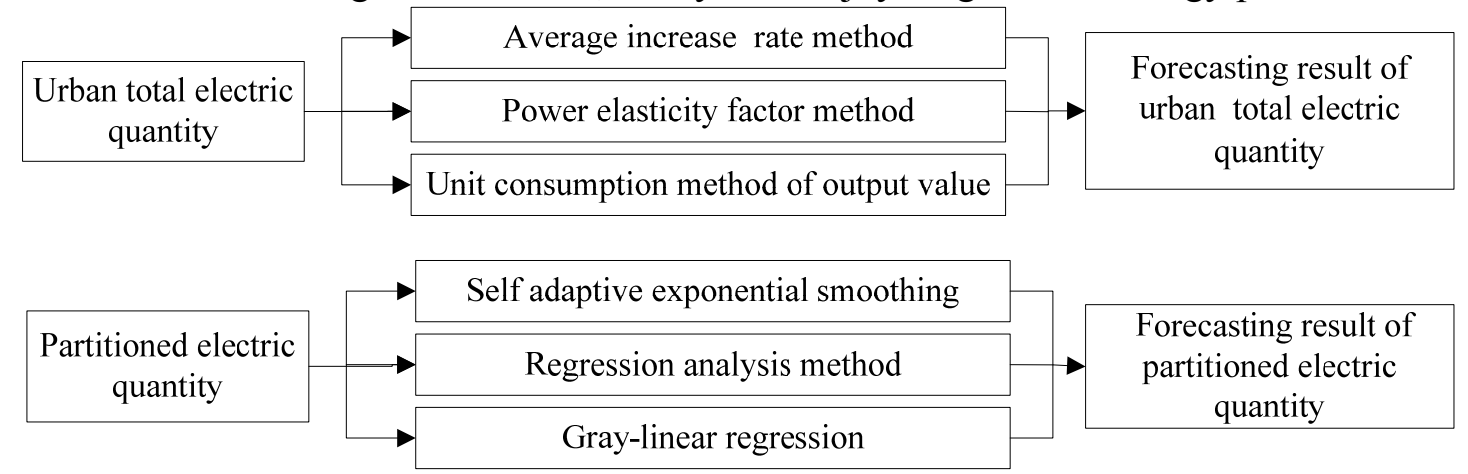

Fig.2 Process of electric quantity forecasting

For electric quantity [4], system employs mathematical methods and economic methods to forecast electric quantity of the urban and partition. Average increase rate method, power elasticity factor method and unit consumption of output value method are used to forecast urban total electric quantity; self-adaptive exponential smoothing, regression analysis method and gray-linear regression method are used to forecast partitioned electric quantity. As shown in figure 2.

For peak load [5], regression analysis, grey model and large customer method are used to forecast urban total peak load and partitioned peak load. As shown in figure 3.

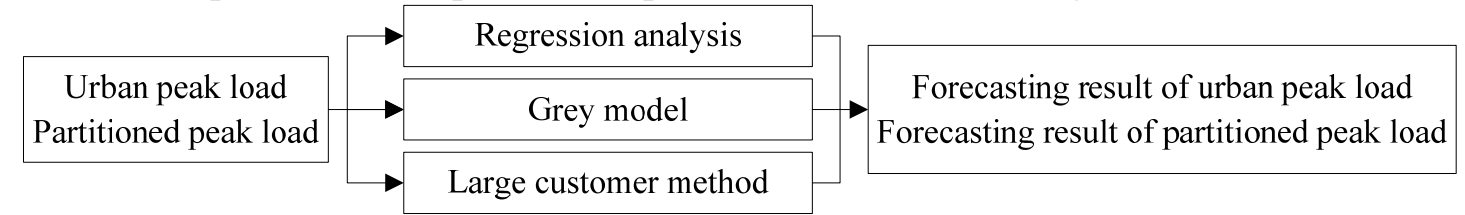

Fig.3. Process of peak load forecasting

For spatial load forecasting [6-7], load density target method based on least square support 
vector machine (LSSVM) is employed. Specific steps are as follows:

(1) Establish index system for load density: a). Combined with the urban planning, categorize load; b). Gather data of relevant factors which affect load density value; c). establish index system for load density.

(2) Cell division;

(3) Screen training samples of LSSVM forecasting model;

(4) Obtain load density of forecasted cell.

Process is shown in figure 4.

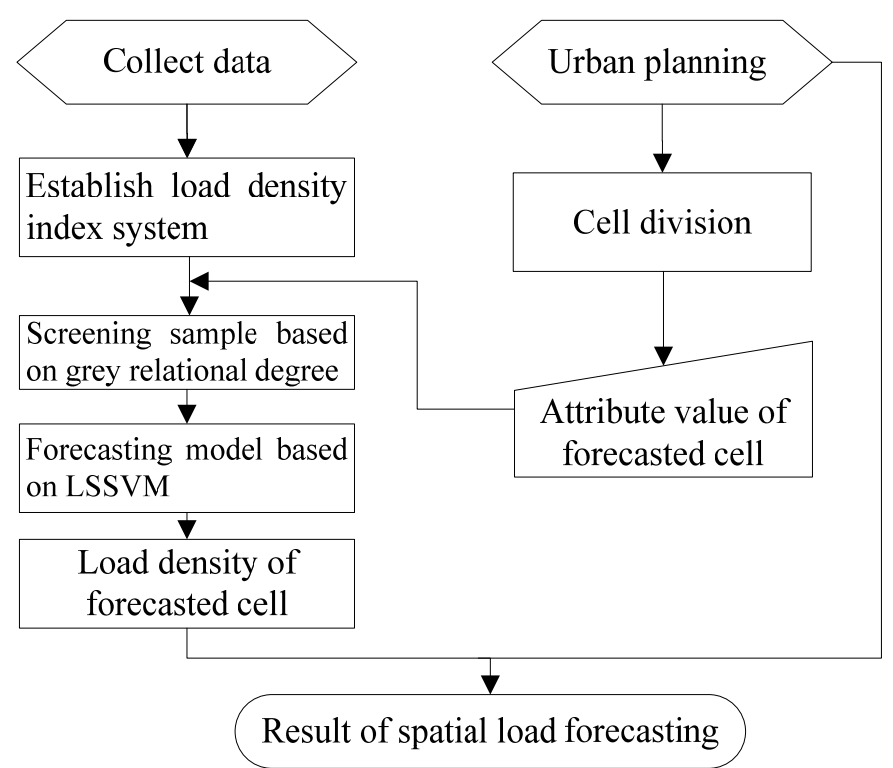

Fig.4 Process of spatial load forecasting

Forecasting results obtained by different methods have certain differences, the system is able to check them with each other, and finally select a reasonable forecasting result as a recommendation. Schematic diagram of checking is shown in figure 5.

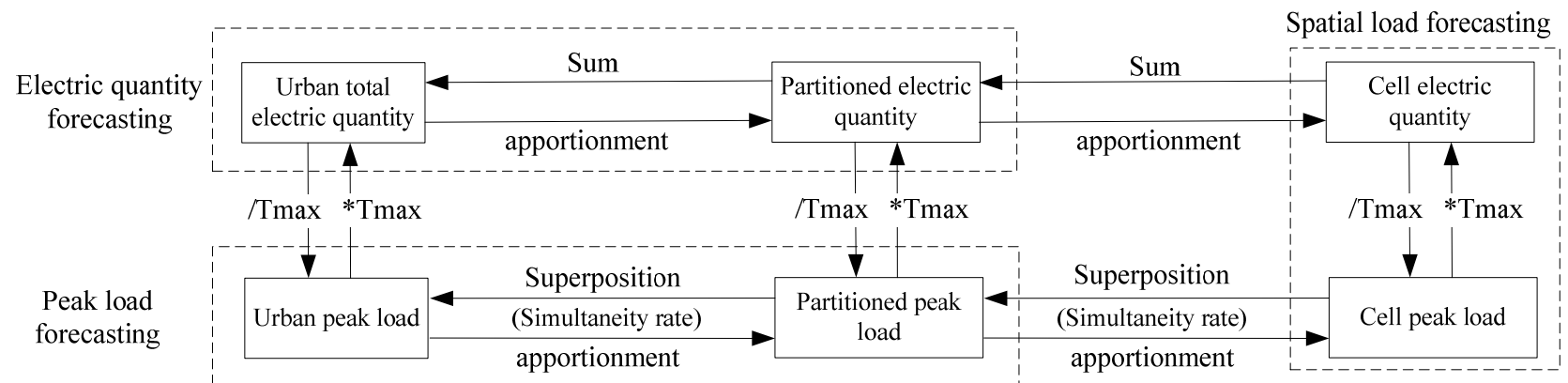

Fig.5 Schematic diagram of power demand forecasting results being checked

A case of $\mathrm{H}$ city is studied in this decision support system used power demand forecasting methods aforementioned. According to the historical load values and the overall urban planning of the city, we get $\mathrm{H}$ city load forecasting result of 13th Five Year distribution network planning as shown in table 1, urban spatial load forecasting result in 2020 target-year as shown in figure 6.

Table 1. Power demand forecasting of H city Unit: Billion kWh; MW; \%; h

\begin{tabular}{|c|c|c|c|c|c|c|c|c|}
\hline year & 2014 (performance) & 2015 & 2016 & 2017 & 2018 & 2019 & 2020 & growth rate \\
\hline electric quantity & 14.7 & 16.3 & 17.8 & 19.6 & 22.0 & 24.3 & 26.1 & $9.95 \%$ \\
\hline peak load & 2480 & 2730 & 3000 & 3350 & 3800 & 4210 & 4600 & $11.01 \%$ \\
\hline $\begin{array}{c}\text { the hours of usage during } \\
\text { peak-load }\end{array}$ & 5924 & 5961 & 5919 & 5857 & 5780 & 5759 & 5684 & -- \\
\hline
\end{tabular}




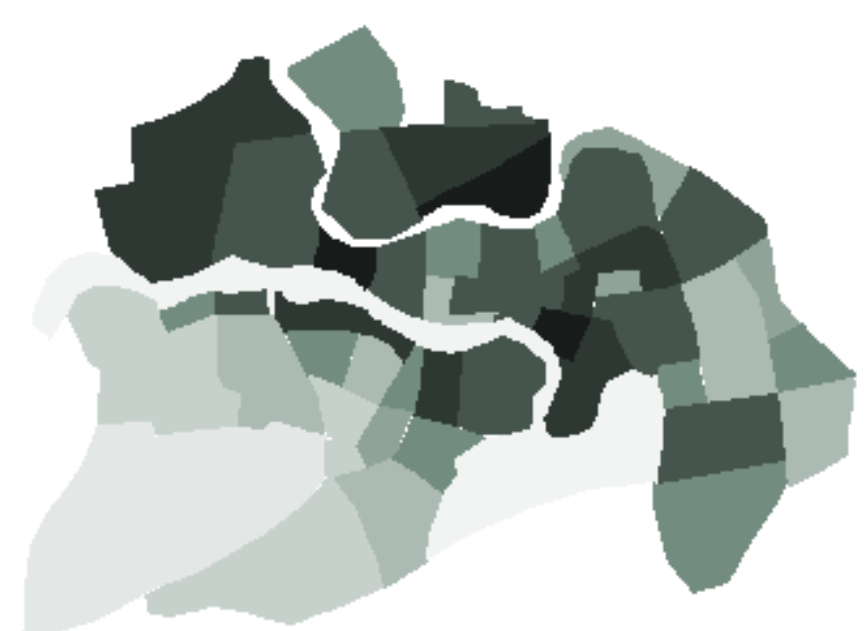

Fig.6 Spatial load forecasting result of H city in 2020 target-year

In Fig.6, varying degrees of color represent different load density area; the deeper color represents the greater load density in the region, which needs to be focused on.

\section{Conclusion}

The decision support system of urban distribution network planning based on electric power demand forecasting is able to manage operating details of the power grid equipment intelligently, also able to present the grid operation state to planners through the visual interface intuitively. Based on load forecasting, system is able to optimize the structure of power supply, and transformer substation location, as well as the arrangement of distribution network planning schemes, thus to improve work efficiency and enthusiasm of the planners, and the economic benefit of power supply enterprise. In addition, reasonable planning obtained by the system is able to reduce the customer premises equipment damages caused by voltage quality problems, and finally provides efficient, safe and reliable electricity for the masses.

\section{References}

[1] C.S. Wang, S.Y. Wang, Y.H. Xie, et al. The complexity and new technology application of urban power distribution system planning. Zhejiang Electric Power,2004,23(1):1-5.

[2] F.Z. Luo, C.S. Wang, J. Xiao, et al. Decision- Making support system for urban distribution network planning of Shanghai. Power System Technology,2009,33(3):79-83.

[3] H.H. Fang, H.Z. Cheng, J.Q. Xin, et al. Indices System of Distribution Network Planning Evaluation. Proceedings of the CSU-EPSA,2013,25(6):106-111.

[4] X. Zhang, G. Wei, M. Zhou, et al. Application of the grey theory in forecasting city annual electricity consumption.Journal of Shanghai University of Electric Power,2002,18(2):9-12.

[5] Yang Hong-tzer, Liang Tian-chyi, Shih Kuang-rong, et al. Power System Yearly Peak Load Forecasting: A Grey System Modeling Approach. IEEE Catalogue, 1995 (3): 261-266.

[6] Willis H L. Spatial electric load forecasting. New York: Marcel Dekker, 2002, 8-20.

[7] B. Xiao, C. Zhou, G. Mu. Review and prospect of the spatial load forecasting methods. Proceedings of the CSEE, 2013,33(25):78-92. 